\title{
THE STRONG AJ CONJECTURE FOR CABLES OF TORUS KNOTS
}

\author{
ANH T. TRAN
}

\begin{abstract}
The AJ conjecture, formulated by Garoufalidis, relates the A-polynomial and the colored Jones polynomial of a knot in the 3 -sphere. It has been confirmed for all torus knots, some classes of two-bridge knots and pretzel knots, and most cabled knots over torus knots. The strong AJ conjecture, formulated by Sikora, relates the A-ideal and the colored Jones polynomial of a knot. It was confirmed for all torus knots. In this paper we confirm the strong AJ conjecture for most cabled knots over torus knots.
\end{abstract}

\section{IntroduCtion}

0.1. The colored Jones polynomial. For a knot $K$ in $S^{3}$ and a positive integer $n$, let $J_{K}(n) \in \mathbb{Z}\left[t^{ \pm 1}\right]$ denote the $n$-colored Jones polynomial of $K$ with zero-framing. The polynomial $J_{K}(n)$ is the quantum link invariant, as defined by Reshetikhin and Turaev [RT], associated to the Lie algebra $s l_{2}(\mathbb{C})$, with the color $n$ standing for the irreducible $s l_{2}(\mathbb{C})$-module $V_{n}$ of dimension $n$. Here we use the functorial normalization, i.e. the one for which the colored Jones polynomial of the unknot $U$ is

$$
J_{U}(n)=[n]:=\frac{t^{2 n}-t^{-2 n}}{t^{2}-t^{-2}}
$$

It is known that $J_{K}(1)=1$ and $J_{K}(2)$ is the usual Jones polynomial [Jo]. The colored Jones polynomials of higher colors are more or less the usual Jones polynomials of parallels of the knot. The color $n$ can be assumed to take negative integer values by setting $J_{K}(-n):=-J_{K}(n)$. In particular, one has $J_{K}(0)=0$.

0.2 . The recurrence ideal and recurrence polynomial. Consider a discrete function $f: \mathbb{Z} \rightarrow \mathcal{R}:=\mathbb{C}\left[t^{ \pm 1}\right]$ and define the linear operators $L, M$ acting on such functions by

$$
(L f)(n):=f(n+1), \quad(M f)(n):=t^{2 n} f(n) .
$$

It is easy to see that $L M=t^{2} M L$, and that $L^{ \pm 1}, M^{ \pm 1}$ generate the quantum torus $\mathcal{T}$, a non-commutative ring with presentation

$$
\mathcal{T}:=\mathcal{R}\left\langle L^{ \pm 1}, M^{ \pm 1}\right\rangle /\left(L M-t^{2} M L\right) .
$$

Let

$$
\mathcal{A}_{K}:=\left\{P \in \mathcal{T} \mid P J_{K}=0\right\},
$$

which is a left-ideal of $\mathcal{T}$, called the recurrence ideal of $K$. It was proved in GL that for every knot $K$, the recurrence ideal $\mathcal{A}_{K}$ is non-zero. An element in $\mathcal{A}_{K}$ is called a recurrence relation for the colored Jones polynomial of $K$.

The ring $\mathcal{T}$ is not a principal left-ideal domain, i.e. not every left-ideal of $\mathcal{T}$ is generated by one element. By adding all inverses of polynomials in $t, M$ to $\mathcal{T}$ one gets a principal left-ideal domain $\tilde{\mathcal{T}}$, c.f. [Ga2]. The ring $\tilde{\mathcal{T}}$ can be formally defined as follows. Let $\mathcal{R}(M)$

2010 Mathematics Classification: Primary 57N10. Secondary 57M25.

Key words and phrases: colored Jones polynomial, A-polynomial, AJ conjecture, torus knot. 
be the fractional field of the polynomial $\operatorname{ring} \mathcal{R}[M]$. Let $\tilde{\mathcal{T}}$ be the set of all Laurent polynomials in the variable $L$ with coefficients in $\mathcal{R}(M)$ :

$$
\tilde{\mathcal{T}}:=\left\{\sum_{j \in \mathbb{Z}} a_{j}(M) L^{j} \mid \quad a_{j}(M) \in \mathcal{R}(M), f_{j}=0 \quad \text { almost everywhere }\right\},
$$

and define the product in $\tilde{\mathcal{T}}$ by $a(M) L^{k} \cdot b(M) L^{l}:=a(M) b\left(t^{2 k} M\right) L^{k+l}$.

The left ideal extension $\tilde{\mathcal{A}}_{K}:=\tilde{\mathcal{T}} \mathcal{A}_{K}$ of $\mathcal{A}_{K}$ in $\tilde{\mathcal{T}}$ is then generated by a polynomial

$$
\alpha_{K}(t ; M, L)=\sum_{j=0}^{d} \alpha_{K, j}(t, M) L^{j},
$$

where $d$ is assumed to be minimal and all the coefficients $\alpha_{K, j}(t, M) \in \mathbb{Z}\left[t^{ \pm 1}, M\right]$ are assumed to be co-prime. That $\alpha_{K}$ can be chosen to have integer coefficients follows from the fact that $J_{K}(n) \in \mathbb{Z}\left[t^{ \pm 1}\right]$. The polynomial $\alpha_{K}$ is defined up to a polynomial in $\mathbb{Z}\left[t^{ \pm 1}, M\right]$. We call $\alpha_{K}$ the recurrence polynomial of $K$.

0.3. The AJ conjecture. The colored Jones polynomials are powerful invariants of knots, but little is known about their relationship with classical invariants like the fundamental group. Inspired by the theory of noncommutative A-ideals of Frohman, Gelca and Lofaro [FGL, Ge] and the theory of $q$-holonomicity of quantum invariants of Garoufalidis and Le GL, Garoufalidis Ga2 formulated the following conjecture that relates the A-polynomial and the colored Jones polynomial of a knot in the 3-pshere.

Conjecture 1. (AJ conjecture) For every knot $K$ in $S^{3},\left.\alpha_{K}\right|_{t=-1}$ is equal to the A-polynomial, up to a polynomial depending on $M$ only.

The $A$-polynomial of a knot was introduced by Cooper et al. [CCGLS; it describes the $S L_{2}(\mathbb{C})$-character variety of the knot complement as viewed from the boundary torus. Here in the definition of the $A$-polynomial, we also allow the factor $L-1$ coming from the abelian component of the character variety of the knot group. Hence the $A$-polynomial in this paper is equal to $L-1$ times the $A$-polynomial defined in [CCGLS].

The AJ conjecture has been confirmed for the trefoil knot and the figure eight knot (by Garoufalidis [Ga2]), all torus knots (by Hikami [Hi], Tran [Tr]), some classes of two-bridge knots and pretzel knots including most double twist knots and $(-2,3,6 n \pm 1)$-pretzel knots (by Le [Le], Le and Tran [LT]), the knot $7_{4}$ (by Garoufalidis and Koutschan [GK]), and most cabled knots over torus knots (by Ruppe and Zhang [RZ]).

0.4. Main result. For a finitely generated group $G$, let $\chi(G)$ denote the $S L_{2}(\mathbb{C})$-character variety of $G$, see [CS]. For a manifold $Y$ we use $\chi(Y)$ also to denote $\chi\left(\pi_{1}(Y)\right)$. Suppose $G=\mathbb{Z}^{2}$. Every pair of generators $\mu, \lambda$ will define an isomorphism between $\chi(G)$ and $\left(\mathbb{C}^{*}\right)^{2} / \tau$, where $\left(\mathbb{C}^{*}\right)^{2}$ is the set of non-zero complex pairs $(M, L)$ and $\tau$ is the involution $\tau(M, L):=\left(M^{-1}, L^{-1}\right)$. For an algebraic set $V$ (over $\mathbb{C}$ ), let $\mathbb{C}[V]$ denote the ring of regular functions on $V$. For example, $\mathbb{C}\left[\left(\mathbb{C}^{*}\right)^{2} / \tau\right]=\mathfrak{t}^{\sigma}$, the $\sigma$-invariant subspace of $\mathfrak{t}:=\mathbb{C}\left[M^{ \pm 1}, L^{ \pm 1}\right]$, where $\sigma\left(M^{k} L^{l}\right):=M^{-k} L^{-l}$.

Let $K$ be a knot in $S^{3}$ and $X=S^{3} \backslash K$ its complement. The boundary of $X$ is a torus whose fundamental group is free abelian of rank two. An orientation of $K$ will define a unique pair of an oriented meridian $\mu$ and an oriented longitude $\lambda$ such that the linking number between the longitude and the knot is zero. The pair provides an identification of $\chi(\partial X)$ and $\left(\mathbb{C}^{*}\right)^{2} / \tau$ which actually does not depend on the orientation of $K$. 
The inclusion $\partial X \hookrightarrow X$ induces an algebra homomorphism

$$
\theta: \mathbb{C}[\chi(\partial X)] \equiv \mathfrak{t}^{\sigma} \longrightarrow \mathbb{C}[\chi(X)]
$$

We call the kernel $\mathfrak{p}$ of $\theta$ the $A$-ideal of $K$; it is an ideal of $\mathfrak{t}^{\sigma}$. The $A$-ideal was first introduced in [FGL]; it determines the $A$-polynomial of $K$. In fact $\mathfrak{p}=\left(A_{K} \cdot \mathfrak{t}\right)^{\sigma}$, the $\sigma$-invariant part of the ideal $A_{K} \cdot \mathfrak{t} \subset \mathfrak{t}$ generated by the $A$-polynomial $A_{K}$.

The involution $\sigma$ acts on the quantum torus $\mathcal{T}$ also by $\sigma\left(M^{k} L^{l}\right)=M^{-k} L^{-l}$. Let $\mathcal{A}_{K}^{\sigma}$ be the $\sigma$-invariant part of the recurrence ideal $\mathcal{A}_{K}$; it is an ideal of $\mathcal{T}^{\sigma}$.

Sikora [Si] formulated the following conjecture that relates the A-ideal and the colored Jones polynomial of a knot in the 3-pshere.

Conjecture 2. (Strong AJ conjecture) Suppose $K$ is a knot in $S^{3}$. Then

$$
\sqrt{\left.\mathcal{A}_{K}^{\sigma}\right|_{t=-1}}=\mathfrak{p}
$$

Here $\sqrt{\left.\mathcal{A}_{K}^{\sigma}\right|_{t=-1}}$ denotes the radical of the ideal $\left.\mathcal{A}_{K}^{\sigma}\right|_{t=-1}$ in the ring $\mathfrak{t}^{\sigma}$.

The strong AJ conjecture was confirmed for the trefoil knot (by Sikora [Si]) and all torus knots (by Tran [Tr]). In this paper we consider the strong AJ conjecture for cabled knots over torus knots. Recall that the set of non-trivial torus knots $T(p, q)$ in $S^{3}$ can be indexed by pairs of relatively prime integers $(p, q)$ satisfying $|p|>q \geq 2$. Also recall that an $(r, s)$-cabled knot over a knot $K$ in $S^{3}$ is the knot which can be embedded in the boundary torus of a tubular neighborhood of $K$ in $S^{3}$ as a curve of slope $r / s$ with respect to the meridian/longitude coordinates of $K$ satisfying $(r, s)=1$ and $s \geq 2$.

Our main result is the following.

Theorem 1. The strong AJ conjecture holds true for each $(r, s)$-cabled knot over each $(p, q)$-torus knot if $r$ is not a number between 0 and pqs.

0.5. Plan of the paper. In Section 1 we prove some properties of the colored Jones polynomial of cabled knots. In Section 2 we prove a result about the relationship between the AJ conjecture and the strong AJ conjecture, and consequently prove one part of Theorem 1, The proof of the other part of Theorem 1 is given in Sections 36 .

0.6. Acknowledgment. We would like to thank X. Zhang for helpful discussions.

\section{The Colored Jones polynomial of CABled knots}

By [Mo, the formula for the colored Jones polynomial of the $(r, s)$-cabled knot $K^{(r, s)}$ over a knot $K$ is given by

$$
J_{K(r, s)}(n)=t^{-r s\left(n^{2}-1\right)} \sum_{j=-\frac{n-1}{2}}^{\frac{n-1}{2}} t^{4 r j(j s+1)} J_{K}(2 s j+1) .
$$

Lemma 1.1. One has

$$
\begin{aligned}
& J_{K^{(r, s)}}(n+2)-t^{-4 r s(n+1)} J_{K^{(r, s)}}(n) \\
= & t^{-2 r s(n+1)}\left(t^{2 r(n+1)} J_{K}(s(n+1)+1)-t^{-2 r(n+1)} J_{K}(s(n+1)-1)\right) .
\end{aligned}
$$


Proof. From Eq. (1.1) we have

$$
\begin{aligned}
& J_{K^{(r, s)}}(n+2)-t^{-4 r s(n+1)} J_{K^{(r, s)}}(n) \\
= & t^{-r s\left((n+2)^{2}-1\right)} \sum_{j=-\frac{n+1}{2}}^{\frac{n+1}{2}} t^{4 r j(s j+1)} J_{K}(2 s j+1)-t^{-4 r s(n+1)} t^{-r s\left(n^{2}-1\right)} \sum_{j=-\frac{n-1}{2}}^{\frac{n-1}{2}} t^{4 r j(j s+1)} J_{K}(2 s j+1) \\
= & t^{-r s\left((n+2)^{2}-1\right)}\left(t^{r(n+1)(s(n+1)+2)} J_{K}(s(n+1)+1)+t^{r(n+1)(s(n+1)-2)} J_{K}(-s(n+1)+1)\right) .
\end{aligned}
$$

The lemma follows since $J_{K}(-s(n+1)+1)=-J_{K}(s(n+1)-1)$.

Lemma 1.2. One has

$$
J_{K^{(r, 2)}}(n+1)+t^{-2 r(2 n+1)} J_{K^{(r, 2)}}(n)=t^{-2 r n} J_{K}(2 n+1) .
$$

Proof. By Eq. (1.1) we have

$$
J_{K^{(r, 2)}}(n+1)=t^{-2 r\left((n+1)^{2}-1\right)} \sum_{k=-\frac{n}{2}}^{\frac{n}{2}} t^{4 r k(2 k+1)} J_{K}(4 k+1) .
$$

Set $k=-\left(j+\frac{1}{2}\right)$. Then

$$
\begin{aligned}
J_{K^{(r, 2)}}(n+1) & =t^{-2 r\left((n+1)^{2}-1\right)} \sum_{j=\frac{n-1}{2}}^{-\frac{n+1}{2}} t^{4 r j(2 j+1)} J_{K}(-(4 j+1)) \\
& =t^{-2 r\left((n+1)^{2}-1\right)}\left(-\sum_{j=-\frac{n-1}{2}}^{\frac{n-1}{2}} t^{4 r j(2 j+1)} J_{K}(4 j+1)+t^{2 r n(n+1)} J_{K}(2 n+1)\right) \\
& =-t^{-2 r(2 n+1)} J_{K^{(r, 2)}}(n)+t^{-2 r n} J_{K}(2 n+1) .
\end{aligned}
$$

This proves Lemma 1.2 .

Remark 1.3. The proofs of Lemmas 1.1 and 1.2 are similar to those of Lemmas 1.1 and 1.5 in [ $\mathrm{Tr}$. See also Propositions 3 and 5 in [Hi], and Lemma 3.1 and Section 6.1 in [RZ].

\section{On the STROng AJ CONJECTURE}

Let $\varepsilon$ be the map reducing $t=-1$.

Lemma 2.1. Suppose the AJ conjecture holds true for a knot $K$ and the A-polynomial $A_{K}$ does not have any non-trivial $M$-factors. Then $\sqrt{\varepsilon\left(\mathcal{A}_{K}^{\sigma}\right)} \subset \mathfrak{p}$.

Proof. Since the AJ conjecture holds true for $K$, we have $\varepsilon\left(\alpha_{K}\right)=f(M) A_{K}$ for some non-zero $f(M) \in \mathbb{C}(M)$. For every $\delta \in \mathcal{A}_{K}$, by [Tr, Lemma 2.5], there exist $g(t, M) \in$ $\mathbb{C}\left[t^{ \pm 1}, M\right]$ and $\gamma \in \mathcal{T}$ such that $\delta=\frac{1}{g(t, M)} \gamma \alpha_{K}$ and $\varepsilon(g) \neq 0$. It follows that

$$
\varepsilon(\delta)=\frac{1}{\varepsilon(g(M))} \varepsilon(\gamma) \varepsilon\left(\alpha_{K}\right)=\frac{1}{\varepsilon(g(M))} \varepsilon(\gamma) f(M) A_{K} .
$$

Let $h=\frac{f(M)}{\varepsilon(g(M))} \varepsilon(\gamma) \in \mathbb{C}(M)\left[L^{ \pm 1}\right]$. Then $\varepsilon(\delta)=h A_{K}$. Since $A_{K}$ does not have any non-trivial $M$-factors, we must have $h \in \mathbb{C}\left[M^{ \pm 1}, L^{ \pm 1}\right]=\mathfrak{t}$. Hence $\varepsilon(\delta) \in A_{K} \cdot \mathfrak{t}$, the ideal of $\mathfrak{t}$ generated by $A_{K}$. It follows that $\varepsilon\left(\mathcal{A}_{K}\right) \subset A_{K} \cdot \mathfrak{t}$ and thus $\varepsilon\left(\mathcal{A}_{K}^{\sigma}\right) \subset\left(A_{K} \cdot \mathfrak{t}\right)^{\sigma}=\mathfrak{p}$. Hence $\sqrt{\varepsilon\left(\mathcal{A}_{K}^{\sigma}\right)} \subset \sqrt{\mathfrak{p}}=\mathfrak{p}$. 
Lemma 2.2. Suppose there exists $P \in \mathcal{A}_{K}^{\sigma}$ such that $\varepsilon(P)=M^{k} L^{l}\left(A_{K}\right)^{2 m}$ for some integers $k, l, m$. Then $\mathfrak{p} \subset \sqrt{\varepsilon\left(\mathcal{A}_{K}^{\sigma}\right)}$.

Proof. It is known that $A_{K}\left(M^{ \pm 1}, L^{ \pm 1}\right)=\eta M^{a} L^{b} A_{K}(M, L)$ where $a, b \in \mathbb{Z}$ and $\eta= \pm 1$, see [CCGLS]. Since $\sigma(P)=P$, we have

$$
M^{k} L^{l}\left(A_{K}\right)^{2 m}=M^{-k} L^{-l}\left(\sigma\left(A_{K}\right)\right)^{2 m}=M^{-k} L^{-l}\left(\eta M^{a} L^{b} A_{K}\right)^{2 m} .
$$

It follows that $k=a m$ and $l=b m$. Hence $\varepsilon(P)=M^{a m} L^{b m}\left(A_{K}\right)^{2 m}$.

If $u \in \mathfrak{p}$ then $u=v A_{K}$ for some $v \in \mathbb{C}\left[M^{ \pm 1}, L^{ \pm 1}\right]$. Since $u=\sigma(u)$, we have $v A_{k}=$ $\sigma(v) \sigma\left(A_{K}\right)=\sigma(v) \eta M^{a} L^{b} A_{K}$ which means that $v=\eta M^{a} L^{b} \sigma(v)$. Hence $M^{-a} L^{-b} v^{2}=$ $\sigma\left(M^{-a} L^{-b} v^{2}\right)$. Let $w=M^{-a} L^{-b} v^{2}$. Then $\sigma(w)=w$. We have

$$
u^{2 m}=v^{2 m}\left(A_{K}\right)^{2 m}=\varepsilon\left(\left(M^{-a} L^{-b} v^{2}\right)^{m} P\right)=\varepsilon\left(w^{m} P\right) \in \varepsilon\left(\mathcal{A}_{K}^{\sigma}\right),
$$

which implies that $u \in \sqrt{\varepsilon\left(\mathcal{A}_{K}^{\sigma}\right)}$. This proves Lemma 2.2.

Let $T=T(p, q)$ be the $(p, q)$-torus knot and $C=T^{(r, s)}$ be the $(r, s)$-cabled knot over $T$. By [RZ], the formula for the A-polynomial of $C$ is given by

$$
A_{C}(M, L)= \begin{cases}(L-1)\left(L^{2}-M^{-2 p q s^{2}}\right)\left(L^{2}-M^{-2 r s}\right) & \text { if } s>2 \text { is odd and } q>2, \\ (L-1)\left(L+M^{-2 p s^{2}}\right)\left(L^{2}-M^{-2 r s}\right) & \text { if } s>2 \text { is odd and } q>2, \\ (L-1)\left(L-M^{-p q s^{2}}\right)\left(L^{2}-M^{-2 r s}\right) & \text { if } s>2 \text { is even, } \\ (L-1)\left(L-M^{-4 p q}\right)\left(L+M^{-2 r}\right) & \text { if } s=2 .\end{cases}
$$

Suppose $r$ is not a number between 0 and pqs. Ruppe and Zhang [RZ] have confirmed the AJ conjecture for the cabled knot $C$. Since the A-polynomial of $C$ does not have any non-trivial $M$-factors, Lemma 2.1 implies that $\sqrt{\varepsilon\left(\mathcal{A}_{C}^{\sigma}\right)} \subset \mathfrak{p}$. Hence to prove the strong AJ conjecture for $C$, we only need to show that

$$
\mathfrak{p} \subset \sqrt{\varepsilon\left(\mathcal{A}_{C}^{\sigma}\right)} .
$$

To prove (2.1) we will apply Lemma 2.2. As in [RZ], the proof of (2.1) is divided into the following 4 cases:

(1) $s$ is odd and $q>2$ (Section 3),

(2) $s$ is odd and $q=2$ (Section 4),

(3) $s>2$ is even (Section 5),

(4) $s=2$ (Section [6).

Moreover, in the case $s>2$ we let $G_{1}(n)=t^{2 r(n+1)} J_{T}(s(n+1)+1)$ and $G_{2}(n)=$ $t^{-2 r(n+1)} J_{T}(s(n+1)-1)$. Then, by Lemma 1.1, we have

$$
t^{2 r s} M^{r s}\left(L^{2}-t^{-4 r s} M^{-2 r s}\right) J_{C}=G_{1}-G_{2} .
$$

The following lemma will be useful in the proof of (2.1).

Lemma 2.3. Suppose $h_{1}(n), \cdots, h_{k}(n) \in \mathcal{R}\left[t^{ \pm 2 n}\right]$. There exists $P \in \mathcal{R}\left[L^{ \pm 1}\right]$ such that $\sigma(P)=P, \varepsilon(P)=\left(L+L^{-1}-2\right)^{m}$ for some $m \geq 1$, and $P h_{i}=0$ for all $i=1, \cdots, k$.

Proof. Suppose $h(n) \in \mathcal{R}\left[t^{ \pm 2 n}\right]$. Write $h(n)=\sum_{j} \lambda_{j} t^{2 k_{j} n}$, where $\lambda_{j} \in \mathcal{R}$ and $k_{j} \in \mathbb{Z}$. Since $t^{2 k_{j} n}$ is annihilated by $L+L^{-1}-t^{2 k_{j}}-t^{-2 k_{j}}, h(n)$ is annihilated by

$$
P_{h}:=\prod_{j}\left(L+L^{-1}-t^{2 k_{j}}-t^{-2 k_{j}}\right) .
$$

Let $P=P_{h_{1}} \cdots P_{h_{n}}$. Then $P$ satisfies the conditions of Lemma 2.3. 
3. CASE $s>2$ IS ODD AND $q>2$

From [Tr, Lemma 1.1] we have $J_{T}(n+2)-t^{-4 p q(n+1)} J_{T}(n) \in \mathcal{R}\left[t^{ \pm 2 n}\right]$, i.e.

$$
\left(L^{2}-t^{-4 p q} M^{-2 p q}\right) J_{T} \in \mathcal{R}\left[M^{ \pm 1}\right] .
$$

Lemma 3.1. For all positive integers $m$, one has

$$
\left(L^{2 m}-t^{-4 p q m^{2}} M^{-2 p q m}\right) J_{T} \in \mathcal{R}\left[M^{ \pm 1}\right] .
$$

Proof. From (3.1) we have $\left(L^{2} M^{2 p q}-t^{4 p q}\right) J_{T} \in \mathcal{R}\left[M^{ \pm 1}\right]$. It follows that

$$
\left(\left(L^{2} M^{2 p q}\right)^{m}-t^{4 p q m}\right) J_{T} \in \mathcal{R}\left[M^{ \pm 1}\right] .
$$

Since $\left(L^{2} M^{2 p q}\right)^{m}=t^{-4 p q m(m-1)} L^{2 m} M^{2 p q m}$, we have $\left(L^{2 m} M^{2 p q m}-t^{4 p q m^{2}}\right) J_{T} \in \mathcal{R}\left[M^{ \pm 1}\right]$. The lemma follows since $L^{2 m} M^{2 p q m}=t^{8 p q m^{2}} M^{2 p q m} L^{2 m}$.

Proposition 3.2. One has

$$
\begin{aligned}
& \left(L^{2} M^{2 p q s^{2}}+L^{-2} M^{-2 p q s^{2}}-t^{4 r-4 p q s}-t^{-4 r+4 p q s+8 p q s^{2}}\right) G_{1} \in \mathcal{R}\left[M^{ \pm 1}\right], \\
& \left(L^{2} M^{2 p q s^{2}}+L^{-2} M^{-2 p q s^{2}}-t^{-4 r+4 p q s}-t^{4 r-4 p q s+8 p q s^{2}}\right) G_{2} \in \mathcal{R}\left[M^{ \pm 1}\right] .
\end{aligned}
$$

Proof. From Lemma 3.1 we have

$$
J_{T}(s(n+3)+1)-t^{-4 p q s(s n+2 s+1)} J_{T}(s(n+1)+1) \in \mathcal{R}\left[t^{ \pm 2 n}\right] .
$$

It follows that $G_{1}(n+2)-t^{4 r-4 p q s(s n+2 s+1)} G_{1}(n) \in \mathcal{R}\left[t^{ \pm 2 n}\right]$. Hence

$$
\left(L^{2}-t^{4 r-4 p q s(2 s+1)} M^{-2 p q s^{2}}\right) G_{1} \in \mathcal{R}\left[M^{ \pm 1}\right],
$$

which implies that $\left(L^{2} M^{2 p q s^{2}}-t^{4 r-4 p q s}\right) G_{1} \in \mathcal{R}\left[M^{ \pm 1}\right]$. Hence

$$
\left(1-t^{-4 r+4 p q s} L^{-2} M^{-2 p q s^{2}}\right)\left(L^{2} M^{2 p q s^{2}}-t^{4 r-4 p q s}\right) G_{1} \in \mathcal{R}\left[M^{ \pm 1}\right] .
$$

The proof for $G_{2}$ is similar.

Let

$$
\begin{aligned}
& P_{1}:=L^{2} M^{2 p q s^{2}}+L^{-2} M^{-2 p q s^{2}}-t^{4 r-4 p q s}-t^{-4 r+4 p q s+8 p q s^{2}}, \\
& P_{2}:=L^{2} M^{2 p q s^{2}}+L^{-2} M^{-2 p q s^{2}}-t^{-4 r+4 p q s}-t^{4 r-4 p q s+8 p q s^{2}} .
\end{aligned}
$$

Note that $\sigma\left(P_{i}\right)=P_{i}$ and $P_{1} P_{2}=P_{2} P_{1}$. By Proposition 3.2, we have $P_{i} G_{i} \in \mathcal{R}\left[M^{ \pm 1}\right]$ for $i=1,2$. Let $P=P_{1} P_{2}$. Then $\sigma(P)=P$ and $P G_{i} \in \mathcal{R}\left[M^{ \pm 1}\right]$.

By Lemma 2.3, we can choose $Q \in \mathcal{R}\left[L^{ \pm 1}\right]$ such that $\sigma(Q)=Q, \varepsilon(Q)=\left(L+L^{-1}-2\right)^{m}$ for some $m \geq 1$, and $Q\left(P G_{i}\right)=0$ for all $i=1,2$.

From (2.2) we have $Q P M^{r s}\left(L^{2}-t^{-4 r s} M^{-2 r s}\right) J_{C}=0$. Let

$$
R:=M^{r s} Q P M^{r s}\left(L^{2}-t^{-4 r s} M^{-2 r s}\right)+M^{-r s} Q P M^{-r s}\left(L^{-2}-t^{-4 r s} M^{2 r s}\right) .
$$

Then $\sigma(R)=R$. Since $\mathcal{A}_{C}$ is invariant under $\sigma$ (by [Ga1]), we have $R J_{C}=0$. Note that

$$
\begin{aligned}
\varepsilon(R) & =\left(L^{2} M^{2 r s}+L^{-2} M^{-2 r s}-2\right) \varepsilon(Q P) \\
& =\left(L^{2} M^{2 r s}+L^{-2} M^{-2 r s}-2\right)\left(L^{2} M^{2 p q s^{2}}+L^{-2} M^{-2 p q s^{2}}-2\right)^{2}\left(L+L^{-1}-2\right)^{m} .
\end{aligned}
$$


Let $k:=\max \{m, 2\}$ and $S:=\left(L^{2} M^{2 r s}+L^{-2} M^{-2 r s}-2\right)^{k-1}\left(L^{2} M^{2 p q s^{2}}+L^{-2} M^{-2 p q s^{2}}-\right.$ $2)^{k-2}\left(L+L^{-1}-2\right)^{k-m}$. We have $\sigma(S)=S$ and

$$
\begin{aligned}
\varepsilon(S R) & =\left(\left(L^{2} M^{2 r s}+L^{-2} M^{-2 r s}-2\right)\left(L^{2} M^{2 p q s^{2}}+L^{-2} M^{-2 p q s^{2}}-2\right)\left(L+L^{-1}-2\right)\right)^{k} \\
& =M^{2(r+p q s) s k} L^{-5 k}\left((L-1)\left(L^{2}-M^{-2 p q s^{2}}\right)\left(L^{2}-M^{-2 r s}\right)\right)^{2 k} .
\end{aligned}
$$

Since $S R \in \mathcal{A}_{C}^{\sigma}$ and $\varepsilon(S R)=M^{2(r+p q s) s k} L^{-5 k}\left(A_{C}\right)^{2 k}$, Lemma 2.2 implies that $\mathfrak{p} \subset$ $\sqrt{\varepsilon\left(\mathcal{A}_{C}^{\sigma}\right)}$. This proves Theorem 1 in the case $s>2$ is odd and $q>2$.

\section{CASE $s>2$ IS ODD AND $q=2$}

From [Tr, Lemma 1.5] we have $J_{T}(n+1)+t^{-2 p(2 n+1)} J_{T}(n) \in \mathcal{R}\left[t^{ \pm 2 n}\right]$, i.e.

$$
\left(L+t^{-2 p} M^{-2 p}\right) J_{T} \in \mathcal{R}\left[M^{ \pm 1}\right] .
$$

Lemma 4.1. When $q=2$, for all positive integers $m$, one has

$$
\left(L^{m}-(-1)^{m} t^{-2 p m^{2}} M^{-2 p m}\right) J_{T} \in \mathcal{R}\left[M^{ \pm 1}\right] .
$$

Proof. From (4.1) we have $\left(L M^{2 p}+t^{2 p}\right) J_{T} \in \mathcal{R}\left[M^{ \pm 1}\right]$. It follows that

$$
\left(\left(L M^{2 p}\right)^{m}-\left(-t^{2 p}\right)^{m}\right) J_{T} \in \mathcal{R}\left[M^{ \pm 1}\right] .
$$

Since $\left(L M^{2 p}\right)^{m}=t^{-2 p m(m-1)} L^{m} M^{2 p m}$, we have $\left(L^{m} M^{2 p m}-(-1)^{m} t^{2 p m^{2}}\right) J_{T} \in \mathcal{R}\left[M^{ \pm 1}\right]$. The lemma follows since $L^{m} M^{2 p m}=t^{4 p m^{2}} M^{2 p m} L^{m}$.

Proposition 4.2. When $q=2$ and $s$ is odd, one has

$$
\begin{aligned}
& \left(L M^{2 p s^{2}}+L^{-1} M^{-2 p s^{2}}+t^{2 r-2 p s(s+2)}+t^{-2 r+2 p s(3 s+2)}\right) G_{1} \in \mathcal{R}\left[M^{ \pm 1}\right], \\
& \left(L M^{2 p s^{2}}+L^{-1} M^{-2 p s^{2}}+t^{-2 r-2 p s(s-2)}+t^{2 r+2 p s(3 s-2)}\right) G_{2} \in \mathcal{R}\left[M^{ \pm 1}\right] .
\end{aligned}
$$

Proof. From Lemma 4.1 we have

$$
J_{T}(s(n+2)+1)+t^{-2 p s(2 s n+3 s+2)} J_{T}(s(n+1)+1) \in \mathcal{R}\left[t^{ \pm 2 n}\right] .
$$

It follows that $G_{1}(n+1)+t^{2 r-2 p s(2 s n+3 s+2)} G_{1}(n) \in \mathcal{R}\left[t^{ \pm 2 n}\right]$. Hence

$$
\left(L+t^{2 r-2 p s(3 s+2)} M^{-2 p s^{2}}\right) G_{1} \in \mathcal{R}\left[M^{ \pm 1}\right],
$$

which implies that $\left(L M^{2 p s^{2}}+t^{2 r-2 p s(s+2)}\right) G_{1} \in \mathcal{R}\left[M^{ \pm 1}\right]$. Hence

$$
\left(1+t^{-2 r+2 p s(s+2)} L^{-1} M^{-2 p s^{2}}\right)\left(L M^{2 p s^{2}}+t^{2 r-2 p s(s+2)}\right) G_{1} \in \mathcal{R}\left[M^{ \pm 1}\right] .
$$

The proof for $G_{2}$ is similar.

By applying Proposition 4.2, we can show that $\mathfrak{p} \subset \sqrt{\varepsilon\left(\mathcal{A}_{C}^{\sigma}\right)}$ as in Section 3 .

\section{CASE $s>2$ IS EVEN}

Proposition 5.1. When $s$ is even, one has

$$
\begin{aligned}
& \left(L M^{p q s^{2}}+L^{-1} M^{-p q s^{2}}-t^{2 r-p q s(s+2)}-t^{-2 r+p q s(3 s+2)}\right) G_{1} \in \mathcal{R}\left[M^{ \pm 1}\right], \\
& \left(L M^{p q s^{2}}+L^{-1} M^{-p q s^{2}}-t^{-2 r-p q s(s-2)}-t^{2 r+p q s(3 s-2)}\right) G_{2} \in \mathcal{R}\left[M^{ \pm 1}\right] .
\end{aligned}
$$


Proof. From Lemma 3.1 we have

$$
J_{T}(s(n+2)+1)-t^{-p q s(2 s n+3 s+2)} J_{T}(s(n+1)+1) \in \mathcal{R}\left[t^{ \pm 2 n}\right] .
$$

It follows that $G_{1}(n+1)-t^{2 r-p q s(2 s n+3 s+2)} G_{1}(n) \in \mathcal{R}\left[t^{ \pm 2 n}\right]$. Hence

$$
\left(L-t^{2 r-p q s(3 s+2)} M^{-p q s^{2}}\right) G_{1} \in \mathcal{R}\left[M^{ \pm 1}\right],
$$

which implies that $\left(L M^{p q s^{2}}-t^{2 r-p q s(s+2)}\right) G_{1} \in \mathcal{R}\left[M^{ \pm 1}\right]$. Hence

$$
\left(1-t^{-2 r+2 p q(s+2)} L^{-1} M^{-2 p s^{2}}\right)\left(L M^{p q s^{2}}-t^{2 r-p q s(s+2)}\right) G_{1} \in \mathcal{R}\left[M^{ \pm 1}\right] .
$$

The proof for $G_{2}$ is similar.

By applying Proposition [5.1, we can show that $\mathfrak{p} \subset \sqrt{\varepsilon\left(\mathcal{A}_{C}^{\sigma}\right)}$ as in Section 3 .

$$
\text { 6. CASE } s=2
$$

In the case $s=2$ we let $G(n)=J_{K}(2 n+1)$. Then, by Lemma 1.2, we have

$$
M^{r}\left(L+t^{-2 r} M^{-2 r}\right) J_{C}=G .
$$

Proposition 6.1. One has

$$
\left(L M^{4 p q}+L^{-1} M^{-4 p q}-1-t^{8 p q}\right) G \in \mathcal{R}\left[M^{ \pm 1}\right] .
$$

Proof. From [Tr, Lemma 1.1] we have

$$
J_{T}(2 n+3)-t^{-8 p q(n+1)} J_{T}(2 n+1) \in \mathcal{R}\left[t^{ \pm 2 n}\right],
$$

i.e. $\left(L-t^{-8 p q} M^{-4 p q}\right) G \in \mathcal{R}\left[M^{ \pm 1}\right]$. It follows that $\left(L M^{4 p q}-1\right) G \in \mathcal{R}\left[M^{ \pm 1}\right]$. Hence

$$
\left(1-L^{-1} M^{-4 p q}\right)\left(L M^{4 p q}-1\right) G \in \mathcal{R}\left[M^{ \pm 1}\right]
$$

The proposition follows.

Let $P=L M^{4 p q}+L^{-1} M^{-4 p q}-1-t^{8 p q}$. Note that $\sigma(P)=P$. By Proposition 6.1 we have $P G \in \mathcal{R}\left[M^{ \pm 1}\right]$. By Lemma 2.3, we can choose $Q \in \mathcal{R}\left[L^{ \pm 1}\right]$ such that $\sigma(Q)=Q$, $\varepsilon(Q)=\left(L+L^{-1}-2\right)^{m}$ for some $m \geq 1$, and $Q(P G)=0$. Eq. (6.1) then implies that $Q P M^{r}\left(L+t^{-2 r} M^{-2 r}\right) J_{C}=0$. Let

$$
R:=M^{r} Q P M^{r}\left(L+t^{-2 r} M^{-2 r}\right)+M^{-r} Q P M^{-r}\left(L^{-1}+t^{-2 r} M^{2 r}\right) .
$$

Then $\sigma(R)=R$. Since $\mathcal{A}_{C}$ is invariant under $\sigma$, we have $R J_{C}=0$. Note that

$$
\varepsilon(R)=\left(L M^{2 r}+L^{-1} M^{-2 r}-2\right)\left(L M^{4 p q}+L^{-1} M^{-4 p q}-2\right)\left(L+L^{-1}-2\right)^{m} .
$$

Let $S:=\left(L M^{2 r}+L^{-1} M^{-2 r}-2\right)^{m-1}\left(L M^{4 p q}+L^{-1} M^{-4 p q}-2\right)^{m-1}$. We have $\sigma(S)=S$ and

$$
\begin{aligned}
\varepsilon(S R) & =\left(\left(L M^{2 r}+L^{-1} M^{-2 r}+2\right)\left(L M^{4 p q}+L^{-1} M^{-4 p q}-2\right)\left(L+L^{-1}-2\right)\right)^{m} \\
& =M^{2(r+2 p q) m} L^{-3 m}\left((L-1)\left(L+M^{-2 r}\right)\left(L-M^{-4 p q}\right)\right)^{2 m} .
\end{aligned}
$$

Since $S R \in \mathcal{A}_{C}^{\sigma}$ and $\varepsilon(S R)=M^{2(r+2 p q) m} L^{-3 m}\left(A_{C}\right)^{2 m}$, Lemma 2.2 implies that $\mathfrak{p} \subset$ $\sqrt{\varepsilon\left(\mathcal{A}_{C}^{\sigma}\right)}$. This proves Theorem 1 in the case $s=2$. 


\section{REFERENCES}

[CCGLS] D. Cooper, M. Culler, H. Gillet, D. Long and P. Shalen, Plane curves associated to character varieties of 3-manifolds, Invent. Math. 118 (1994), pp. 47-84.

[CS] M. Culler and P. Shalen, Varieties of group representations and splittings of 3-manifolds, Ann. of Math. (2) 117 (1983), no. 1, 109-146.

[FGL] C. Frohman, R. Gelca, and W. Lofaro, The A-polynomial from the noncommutative viewpoint, Trans. Amer. Math. Soc. 354 (2002), no. 2, 735-747.

[Ga1] S. Garoufalidis, Difference and differential equations for the colored Jones function, J. Knot Theory and Its Ramifications 17 (2008), no. 4, 495-510.

[Ga2] S. Garoufalidis, On the characteristic and deformation varieties of a knot, Proceedings of the Casson Fest, Geom. Topol. Monogr., vol. 7, Geom. Topol. Publ., Coventry, 2004, 291-309 (electronic).

[Ge] R. Gelca, On the relation between the A-polynomial and the Jones polynomial, Proc. Amer. Math. Soc. 130 (2002), no. 4, 1235-1241.

[GK] C. Koutschan and S. Garoufalidis, Irreducibility of q-difference operators and the knot $7_{4}$, Algebr. Geom. Topol. (to appear).

[GL] S. Garoufalidis and T. Le, The colored Jones function is q-holonomic, Geom. Topol. 9 (2005), 1253-1293 (electronic).

[Hi] K. Hikami, Difference equation of the colored Jones polynomial for torus knot, Internat. J. Math. 15 (2004), no. 9, 959-965.

[Jo] V. Jones, Hecke algebra representations of braid groups and link polynomials, Ann. of Math. (2) 126 (1987), 335-388.

[Le] T. Le, The colored Jones polynomial and the A-polynomial of knots, Adv. Math. 207 (2006), no. $2,782-804$.

[LT] T. Le and A. Tran, On the AJ conjecture for knots, preprint 2012, arXiv:1111.5258.

[Mo] H. Morton, The coloured Jones function and Alexander polynomial for torus knots, Math. Proc. Cambridge Philos. Soc. 117 (1995), no. 1, 129-135.

[RT] N. Yu. Reshetikhin and V. Turaev, Ribbon graphs and their invariants derived from quantum groups, Commun. Math. Phys. 127 (1990), 1-26.

[RZ] D. Ruppe and X. Zhang, The AJ conjecture and cabled knots over torus knots, preprint 2014, arXiv:1403.1858,

[Si] A. Sikora, Quantizations of character varieties and quantum knot invariants, preprint 2008, arXiv:0807.0943,

[Tr] A. Tran, Proof of a stronger version of the AJ conjecture for torus knots, Algebr. Geom. Topol. 13 (2013), no. 1, 609-624.

Department of Mathematics, The Ohio State University, Columbus, OH 43210, USA

E-mail address: tran.350@osu.edu 